\title{
Manifestaciones cutáneas de las intoxicaciones por arsénico, plomo, mercurio y plata
}

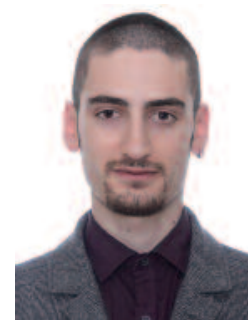

\section{Davide Luordo}

Estudiante de 5.ํㅜㅇ curso de Medicina. Hospital Universitario

12 de Octubre.

Universidad Complutense de Madrid.

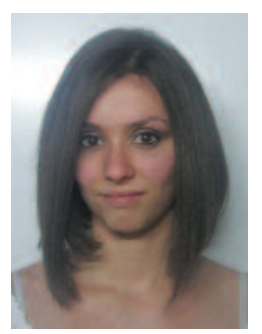

Deborah Forrester Zapata

Estudiante de 5.. curso de Medicina. Hospital Universitario 12 de Octubre.

Universidad Complutense de Madrid.

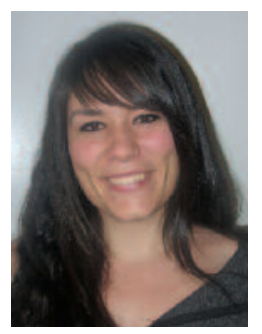

\section{Sara Lorenzo Mouronte}

Estudiante de 5. ${ }^{\circ}$ curso de Medicina. Hospital Universitario 12 de Octubre. Universidad Complutense de Madrid.

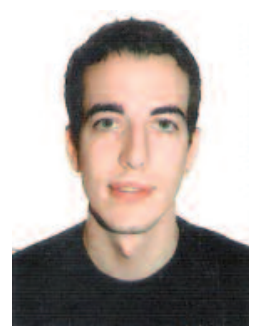

\section{Francisco Encabo Fernández}

Estudiante de $5 .^{\circ}$ curso de Medicina. Hospital Universitario 12 de Octubre. Universidad Complutense de Madrid.

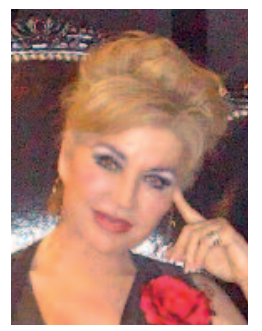

Aurora Guerra-Tapia

Profesora titular de Dermatología. Hospital Universitario 12 de Octubre. Universidad Complutense de Madrid.

\section{RESUMEN}

Las intoxicaciones por metales, tanto en sus fases agudas como crónicas, pueden determinar la aparición de signos dermatológicos característicos, signos que son objeto de estudio de esta revisión bibliográfica que hemos querido relacionar con distintos eventos históricos. Nos hemos centrado en una serie de compuestos por su especial relevancia desde el punto de vista tanto epidemiológico, ya que representan las intoxicaciones por metales que más inciden en nuestro medio, como dermatológico, por sus frecuentes y evidentes manifestaciones mucocutáneas.

Palabras clave: arsénico, líneas de Mees, hiperqueratosis palmoplantar, plomo, saturnismo, ribete de Burton, mercurio, hidrargiria, eretismo mercurial, ribete de Gilbert, síndrome de baboon, plata, argiria, blue men.

\section{ABSTRACT}

Metal poisonings, both acute and chronic forms, can determin the onset of specific dermatologic signs that are de subject of study of this literature review, which we have wanted to relate with diverse historical events. We have focused on a series of compounds due to their relevance both from the epidemiological point of view, as they represent the most incident metal poisoning in our environment, and dermatological point of view, because of their frecuent and obvious mucocutaneous manifestations.

Keywords: arsenic, Mees' lines, palmoplantar hyperkeratosis, lead, saturnism, Burton line, mercury, hydrargyria, erethism, Gilbert line, baboon syndrome, silver, blue men.

\section{HISTORIA}

Es difícil definir en qué momento de la historia el hombre comenzó a interesarse por el mundo de los tóxicos, ya que estas sustancias, o bien sus efectos, 
parecen haber estado presentes en todas las civilizaciones desde tiempos inmemoriales, habiéndose introducido de una u otra forma en nuestros conocimientos, ya sea por estudios científicos o con intenciones lúdicas, religiosas o incluso criminales.

Remontándonos al año 1500 a. C., ya en el papiro de Ebers se recogían las primeras referencias escritas explícitas sobre venenos y metales tóxicos como el plomo. Posteriormente, en la Theriaca, Nicandro de Colofón (204-135 a. C.) menciona ya tóxicos como el arsénico o el mercurio y la clínica que producen, mientras que en la Alexipharmaka se indica cómo contrarrestalos ${ }^{1}$.

Existen también referencias sobre metales pertenecientes a la época del Imperio romano, entre las que destaca el saturnismo, descrito como enfermedad de trabajadores de las minas y fundiciones de plomo (el trabajo de minero se equiparaba a la pena de muerte y se reservaba a los peores criminales).

Theophrastus Phillippus Aureolus Bombastus von Hohenheim, más conocido como Paracelso, creció en una región minera y fue familiarizándose con las propiedades de los metales y sus compuestos que, según él, eran superiores a los remedios de hierbas utilizadas por los médicos ortodoxos.

A finales del siglo XIX, el desarrollo de la toxicología permitió configurar una nueva mentalidad interesada en descubrir las causas de las enfermedades, entendiendo la enfermedad como el resultado de la acción de agentes externos, incluidos los tóxicos.

Actualmente, los estudios en el campo de la toxicología se centran principalmente en su implicación en la medicina del trabajo en el estudio de enfermedades laborales producidas por tóxicos ${ }^{2}$.

Enunciando los principios básicos de la toxicología ${ }^{3,4}$, Paracelso afirmó: «dosis sola facit venenum», con la intención de remarcar el principio fundamental de que cualquier sustancia, administrada en determinadas dosis, puede ejercer efec- tos positivos o negativos en el organismo que pueden llegar a ser nefastos ${ }^{5}$.

Con ello es sencillo entender que, efectivamente, cualquier elemento de la tabla periódica, y cualquier compuesto en general, puede comportarse como veneno si se administra en dosis suficientes. De todos los elementos incluidos en las categorías de metales y metaloides, atendiendo a criterios de relevancia histórica y epidemiológica y apoyándonos en lo llamativas que pueden llegar a parecer determinadas manifestaciones de las intoxicaciones por estos elementos, hemos estructurado este estudio como una breve revisión bibliográfica sobre las manifestaciones dermatológicas de las intoxicaciones por arsénico, plomo, mercurio y plata, destacando algunos hechos históricos protagonizados por ellos.

\section{ARSÉNICO}

El arsénico es un metaloide relativamente común en la naturaleza, con un contenido en la corteza terrestre de $5 \mathrm{~g}$ por tonelada, aunque en determinadas zonas (como Irán o Argentina) puede ser mayor, lo que puede dar lugar a fenómenos de contaminación de las aguas y derivar en arsenicismos endémicos.

Presenta un color gris metálico en condiciones normales, aunque también puede aparecer en forma de arsénico amarillo y negro. Se encuentra en su mayor parte formando compuestos (sulfuros, óxidos, arseniuros, sulfoarseniuros y arsenitos). Todas las formas pueden ser tóxicas en mayor o menor medida, aunque las que presentan una mayor toxicidad son: los óxidos y los compuestos sulfurados, que al ser quemados emiten $\mathrm{As}_{2} \mathrm{O}_{3} \mathrm{y}$ $\mathrm{SO}_{2}$, lo que supone un importante riesgo de exposición respiratoria, y la arsina $\left(\mathrm{AsH}_{3}\right)$, que se descompone por la acción de la luz en arsénico negro $\left(\mathrm{As}_{4} \mathrm{O}_{6}\right)$, y por el calor en arsénico metálico.

\section{Aplicaciones}

Actualmente, las principales aplicaciones del arsénico son: como preservador de la madera; en elec- 
trónica, como semiconductor en circuitos integrados y en la construcción de diodos láser y LED (light-emitting diod); como insecticida y herbicida; como pigmento; en la pirotecnia; en la fabricación de vidrio, y en la metalurgia, dado que el arsénico modifica las características físicas del plomo y del cobre, endureciéndolas, por lo que se emplea para la fabricación de perdigones, baterías y cubiertas de cables.

Además, clásicamente se ha utilizado el arsénico como veneno, mientras que en la medicina occidental su finalidad era terapéutica; un ejemplo de ello es la solución de Fowler ${ }^{6,7}$ (que contenía ácido arsenioso y carbonato de potasio, que dan lugar a $\mathrm{KH}_{2} \mathrm{AsO}_{3}$ ), usada para el tratamiento del paludismo, el cólera, la sífilis y el liquen erosivo de mucosas (fig. 1), entre otras enfermedades. También se utilizaban las píldoras asiáticas (que contenían ácido arsenioso, pimienta negra pulverizada, goma arábiga pulverizada y agua) para tratar el herpes y la lepra.

El uso de compuestos inorgánicos de arsénico está prohibido en España desde 1983, aunque algunos médicos homeópatas siguen utilizándolos, lo que constituye una importante fuente de intoxicaciones.

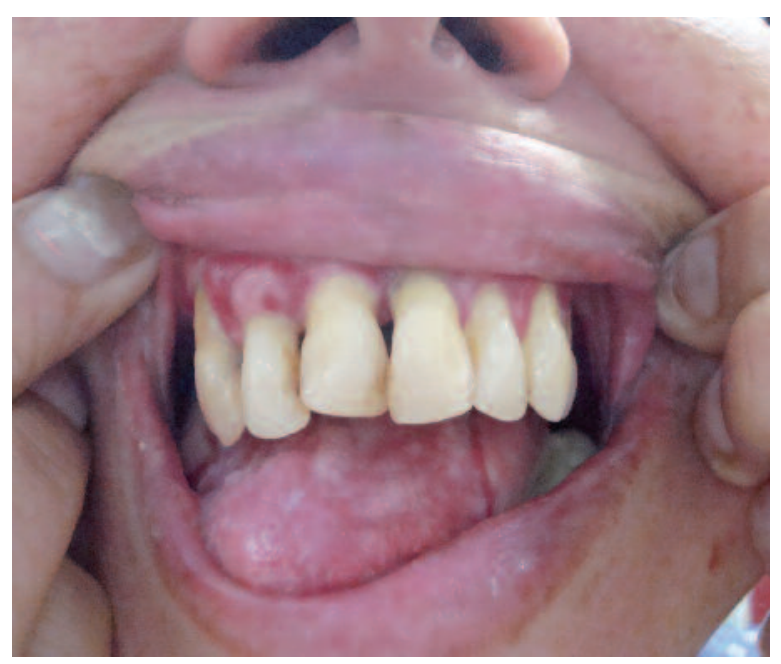

Figura 1. Liquen erosivo de mucosas. Hace décadas se trataban con licor arsenical de Fowler, en dosis creciente y decreciente, una única vez en la vida del paciente.

\section{Clínica}

La clínica varía en función del tiempo de exposición y la vía de entrada en el organismo. Puede ser por vía digestiva (sobre todo en forma de sales y compuestos oxidados), respiratoria (sobre todo la arsina) y raramente dérmica.

Concentraciones en sangre superiores a $3 \mu \mathrm{g} / \mathrm{dl}$ se consideran patológicas, y dosis mayores de $120 \mathrm{mg}$ de $\mathrm{As}_{2} \mathrm{O}_{3}$ se consideran mortales para un adulto.

En general, la clínica puede clasificarse en efectos locales, intoxicaciones agudas e intoxicaciones crónicas.

Entre los efectos locales están la irritación cutánea, con eritema, eccema o úlceras, y la conjuntival, que abarca desde sequedad hasta ulceraciones, llegando a aparecer perforación del tabique nasal y alteraciones de cuerdas vocales.

Las intoxicaciones agudas dan lugar a síntomas gastrointestinales, cefalea, vértigos, sensación de debilidad, dolor torácico por neumonitis si se inhala y alteraciones hemodinámicas a dosis altas (principal causa de muerte), con parálisis capilar, taquicardia y caída de la presión arterial. Si el enfermo sobrevive a las primeras 24 horas, se produce una mejoría relativa, pero aparece hemólisis, insuficiencia hepática y renal e ictericia. Además, durante el periodo de recuperación puede aparecer polineuritis mixta, afectación del sistema vestibular y neuritis del nervio óptico.

Por último, las intoxicaciones crónicas producen alteraciones a múltiples niveles.

En piel y faneras aparece una pigmentación bronceada difusa o en gotas de lluvia con mezcla de máculas hipopigmentadas e hiperpigmentadas (bronceamiento arsenical) y pequeñas pápulas hiperqueratósicas y simétricas en palmas de las manos, plantas de los pies y orejas. Las uñas presentarán unas estriaciones tranversales translúcidas (líneas de Mess). Años después de la exposición pueden aparecer epiteliomas (fig. 2).

En el sistema nervioso periférico aparecen neuropatías periféricas mixtas que se inician en los miembros y avanzan centrípetamente, lo que deriva en parestesias, falta de sensibilidad en 


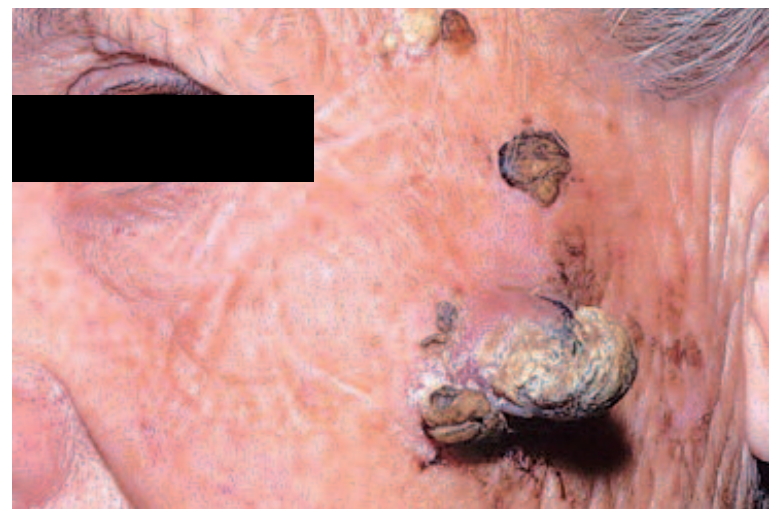

Figura 2. Los epiteliomas múltiples son frecuentes en las intoxicaciones crónicas por arsénico, como el cuerno cutáneo y múltiples queratosis actínicas de la imagen.

guante, parálisis dolorosa y alteraciones de vista y olfato.

Entre otras manifestaciones que hay que destacar están la insuficiencia renal, la disfunción hepática y las alteraciones cardiovasculares, además de tumores, sobre todo dérmicos y labiales, hepáticos y pulmonares.

Como ejemplo de la amplia presencia del arsénico a lo largo de la historia hemos querido rescatar la muerte de Napoleón Bonaparte. Mucho se ha especulado sobre la causa de su fallecimiento, pudiendo diferenciar dos teorías fundamentalmente: cáncer gástrico e intoxicación por arsénico.

La teoría del cáncer gástrico se ve apoyada por la similitud clínica y los antecedentes familiares: el hecho de que durante su largo padecimiento presentara dolor en la región epigástrica y continuos eructos, así como tos seca que ocasionaba vómitos y un profundo abatimiento e inapetencia. Además, según Antommarchi, su médico personal, «el emperador había enflaquecido tan considerablemente [...], que no abulta la cuarta parte que antes». Pero eso no es todo, ya que en la descripción de la autopsia, comenta que «casi toda la superficie interna [del estómago] estaba ocupado por una úlcera cancerosa ${ }^{8}$.

Sin embargo, ¿es esto compatible con una intoxicación crónica por arsénico? Lo es, ya que el arsénico supuestamente administrado y el cloru- ro de mercurio que el doctor Antommarchi le pautaba, pudieron combinarse y producir cianuro de mercurio, sustancia que provoca corrosión en el aparato digestivo. Vale la pena aclarar que en los mechones del emperador se encontró cianuro ${ }^{9}$.

Y por si no fuera suficiente con la polémica entre cáncer gástrico e intoxicación por arsénico, existe también el debate de la intencionalidad contra el accidente.

Para confirmar la hipótesis del envenenamiento se recurrió a un mechón de cabello del emperador: todos los análisis coincidieron en mostrar niveles muy altos de arsénico. Sin embargo, esto también pudo haber ocurrido debido a que las aguas de la isla de Santa Elena, donde permaneció exiliado durante 6 años, contenían concentraciones elevadas de arsénico. Pero el análisis del cabello de la amante de Napoleón en su exilio demostró una concentración arsenical casi normal. ¿Puede ser que a Napoleón lo estuviesen envenenando? Merece la pena destacar que cuando se exhumó el cadáver en 1840 el cuerpo estaba muy bien conservado. ¿Y cuál es uno de los productos que los taxidermistas emplean para la conservación de los cadáveres? Justamente, el arsénico. Ahora bien, es curioso que, tal como se afirmó, «el cadáver no había podido ser embalsamado por falta de las sustancias necesarias» ${ }^{8}$.

Así pues, la polémica persiste.

\section{PLOMO (SATURNISMO)}

El plomo es un metal abundante en la corteza terrestre. Su mineral más frecuente es la galena $\left(\mathrm{PbS}_{2}\right)$, aunque no da lugar a toxicidad por fuentes naturales ${ }^{3,4}$. Se emplea en la mayor parte de sus aplicaciones en forma de aleación con otros metales. Es un metal pesado y tóxico, y la intoxicación por plomo se denomina saturnismo o plumbosis.

\section{Aplicaciones}

Históricamente, el plomo se ha utilizado en múltiples áreas, como la construcción de cañerías, la 
fabricación de contenedores de alimentos, aditivos de vinos, alambiques y en muchos elementos de la vida cotidiana. Esto ha supuesto una fuente muy importante de intoxicaciones, pero en la actualidad sus usos han sido restringidos a antidetonante de gasolina, baterías eléctricas (su producción y manipulación son causa de múltiples intoxicaciones), pinturas, pirotecnia, forros para cables, tubos, hojas y chapas metálicas, fabricación de vidrios y material de soldadura. En los últimos años se ha prestado especial atención al impacto ambiental de los perdigones empleados en la caza. Además, clásicamente se ha utilizado de muy diversas formas en la terapéutica, y especialmente durante el siglo XVIII ${ }^{10}$.

Las múltiples aplicaciones del plomo podrían haber influido en la muerte de Ludwig van Beethoven. Se postulan varias teorías sobre la muerte del famoso músico y compositor. La más aceptada de ellas explica su muerte como consecuencia de una enfermedad hepática de larga evolución debida al abuso del alcohol y a hepatitis, lo que se ve apoyado por el hallazgo en su autopsia de cirrosis y atrofia hepática. Aun así, en el análisis de su cabello se han encontrado niveles excesivamente altos de plomo (algunos estudios indican que hasta cien veces por encima de los valores normales). Un estudio más reciente llevado a cabo por el doctor Christian Reiter intentó medir la distribución del plomo a lo largo de un único cabello para conseguir un «diario químico» de los últimos 4 meses de su vida, relacionando las fechas de los picos de plomo con eventos sucedidos en los días previos. Utilizando este método, el doctor describió cinco picos de plomo en el pelo de Beethoven: el primero de ellos parece estar relacionado temporalmente con la medicación que tomó para una neumonía que contrajo el 5 de diciembre de 1826, mientras que los otros cuatro parecen corresponderse con las paracentesis que se le realizaron los días 20 de diciembre de 1826 y 8 de enero y 2 y 27 de febrero de 1827, lo que sugiere, por tanto, que la herida de la punción pudiera haber sido cubierta con un ungüento o masilla que contuviese plomo, frecuentes en la época. Aun así, esta teoría ha sido muy criticada, dado que el paso del plomo al cabello no se produce en forma de picos tan marcados, sino que se acumula de forma más lenta y constante. Además, el Dr. Wawruch, que asistió a Beethoven durante estas fechas, estaba al tanto de los efectos nocivos del plomo, por lo que parece poco probable que usase altas dosis en su medicación y que cubriese con él sus heridas, dado que según escritos de la época el médico ordenó «mantener la herida meticulosamente seca para evitar infección».

De este modo, quedaría por determinar si, efectivamente, el plomo encontrado en su cabello fue administrado al músico y es resultado de altas concentraciones en sangre o, si por el contrario, es resultado de una contaminación exógena, dado que el pelo es particularmente susceptible a la absorción de plomo exógeno al tener muchos sitios de unión para este en las queratinas que lo forman, y además es imposible distinguir el plomo exógeno del endógeno en las muestras ${ }^{11}$.

\section{Clínica}

El plomo ${ }^{3,4}$ se puede absorber por cualquier vía y actúa en múltiples tejidos; destaca la acción sobre el músculo liso (estimula la contracción mantenida) y la interacción con una serie de sistemas enzimáticos con grupos tiol. Su eliminación es por vía renal y por las heces, y sus dosis tóxicas son muy variables: se estima que la absorción diaria de 1-2 mg de plomo produce saturnismo en pocos meses.

Las manifestaciones de una intoxicación aguda (poco frecuentes en nuestros días) son vómitos con sialorrea, sabor metálico, dolor abdominal, estreñimiento, necrosis tubular, hepatopatía y coma. Sin embargo, es más característica la intoxicación crónica (saturnismo), ya que se producen efectos en múltiples localizaciones. De esta forma, a nivel oral encontramos una acumulación de plomo en forma de sulfuro negro a causa de su eliminación en la saliva, lo que deriva en tatuajes mucosos que también pueden aparecer en las 
mejillas e incluso en la lengua, pudiendo acompañarse de estomatitis. Es típica la aparición del ribete de Burton, un ribete de color gris azulado en la cara externa de la encía superior junto a la raíz de los dientes, más acusado a nivel de los molares $^{12,13}$.

La afectación del músculo liso a nivel gastrointestinal provocará estreñimiento en un comienzo, seguido de diarrea, úlceras y «cólicos saturninos».

En el aparato vascular se producirán espasmos que, junto con el depósito de plomo en los riñones al eliminarse, producirán una lesión tubular.

Desde el punto de vista hematológico aparece anemia microcítica hipocrómica con punteado basófilo en los hematíes.

En el sistema nervioso puede provocar neuropatía periférica motora, y a nivel del sistema nervioso central aparece encefalopatía saturnina, con alteraciones del aprendizaje, irritabilidad, letargia, fatiga, trastornos del sueño, cefalea, ataxias y trastornos del habla, que puede derivar en episodios convulsivos y demencia.

En huesos y articulaciones se produce acumulación de plomo, y puede aparecer seudogota saturnina con artralgia saturnina ${ }^{14}$.

\section{MERCURIO}

El mercurio ${ }^{3,4}$ es un metal pesado que a temperatura ambiente es un líquido inodoro. Su nombre original, hidrargiro, y su símbolo químico derivan del griego hydrargyros (hydros, agua y argiros, plata). Esta denominación está en desuso, y el término mercurio se utiliza en honor al homónimo dios romano, con el que se le comparó por su movilidad.

Los efectos tóxicos del mercurio aparecen descritos ya en la Edad Media. Son, además, típicos de intoxicaciones en determinadas profesiones, como la de sombrerero (se utilizaba en la fabricación de fieltro y como pigmento, siendo el responsable del denominado eretismo mercurial), o en determinados pacientes como los sifilíticos, ya que, antes de la aparición de la penicilina, el tratamiento con mercurio de la sífilis era tan común que se llegó a popularizar la frase «una noche con Venus, toda la vida con Mercurio».

\section{Aplicaciones}

Los usos del mercurio han ido disminuyendo con el tiempo, y en la actualidad sus principales fuentes de exposición son la minería (el cinabrio libera mercurio metálico), la agricultura (antisépticos y tratamiento de madera y grano), la fabricación de termómetros, barómetros, termostatos, trompas de vacío, material de odontología (amalgama dental), tubos fluorescentes, lámparas de mercurio, baterías secas de larga duración, catalizadores y pigmentos y la terapéutica dermatológica (sobre todo el mercurio amoniacal, que se ha utilizado como antiséptico y antipsoriásico).

\section{Clínica}

La intoxicación por mercurio (hidrargiria, hidrargirismo o mercurialismo), dependiendo de las modalidades de exposición, puede clasificarse en aguda o crónica.

La intoxicación aguda se produce normalmente por inhalación de vapores o ingesta de compuestos mercúricos. En la etapa inicial aparece inflamación mucosa (bronquitis, neumonitis, gastritis, etc.) que posteriormente revierte y se sigue de una fase sistémica de predominio digestivo con colitis ulcerohemorrágica y en ocasiones vómitos hemorrágicos incoercibles y shock, siendo típica la intensa sialorrea espumosa. Aproximadamente hacia el segundo o tercer día aparece una estomatitis mercurial, y también pueden aparecer eritemas escarlatiniformes acompañados a menudo de adenopatías, sobre todo en pliegues y región periumbilical.

Por último, se produce un cuadro de insuficiencia renal anúrica por necrosis tubular, que puede derivar en la muerte en un período de 812 días (aunque se puede producir en 24 horas en caso de shock grave).

La intoxicación crónica es típica de profesionales expuestos y puede producirse por deriva- 
dos orgánicos o por mercurio elemental (vapor) y compuestos inorgánicos; esta última es la más frecuente. Se caracteriza por presentar una fase inicial de impregnación, con síntomas generales inespecíficos, y una fase de intoxicación, propiamente dicha, que cursa con alteraciones a múltiples niveles.

En el aparato digestivo destaca la estomatitis mercurial con intensa sialorrea, encías enrojecidas y ligeramente hipertróficas, y en ocasiones úlceras, que pueden complicarse con pérdida de piezas dentarias o necrosis maxilar. En las encías puede aparecer un ribete oscuro gris-azulado debido a la formación de sulfuro de mercurio por fermentación bacteriana denominado lisere hidrargínico de Gilbert, que se diferencia del ribete de Burton por ser más ancho. Los dientes pueden adquirir un color pardusco (diente mercurial de Letuelle) y el paciente nota un sabor metálico constante y molesto, acompañado de aliento fétido ${ }^{15}$.

En el sistema nervioso aparece un cuadro inicial psiquiátrico que se corresponde con el eretismo mercurial (irritabilidad, tristeza, ansiedad, insomnio, temor, pérdida de memoria, excesiva timidez, cambios de humor, etc.), seguido de un cuadro neurológico cuyo síntoma principal es el temblor intencional, variable y por ondas, de comienzo en cara y dedos de las manos, que se intensifica con la excitación y desaparece con el sueño, tal como viene descrito en el personaje del sombrerero del libro Alicia en el país de las maravillas, de Lewis Caroll ${ }^{16}$ y representado en la adaptación cinematográfica de Disney de 1951.

Por último, otras alteraciones remarcables son las producidas a nivel renal y las dermatológicas. Destaca la acrodinia en intoxicaciones por sales de mercurio inorgánico, que cursa, entre otros, con descamación y color rosado en mejillas, palmas y plantas.

También se ha descrito la aparición del cuadro denominado síndrome de baboon o del babuino, una dermatitis de contacto sistémica que cursa con una erupción maculopapular pruriginosa, confluente en el área de los glúteos y en las flexuras

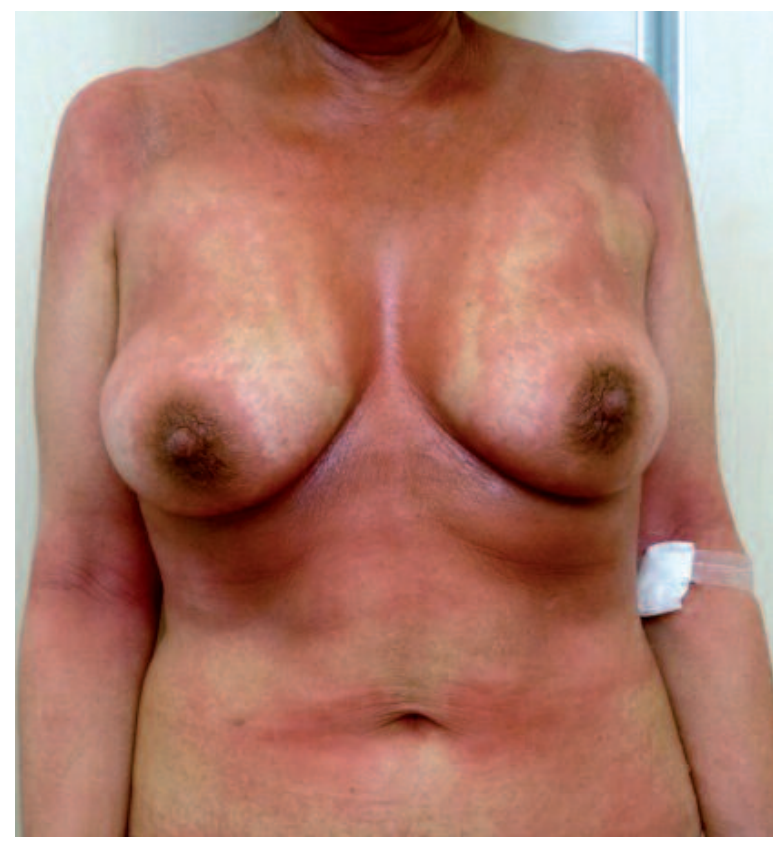

Figura 3. Exantema por contacto sistémico en el síndrome de baboon. (Cortesía de la Dra. Lidia Maroñas.)

mayores (fig. 3), que puede aparecer horas o hasta 5 días después del contacto con ampicilina, níquel y mercurio ${ }^{17,18}$.

\section{PLATA}

Elemento químico de número atómico 47 y masa atómica 107,87; es un metal de color blanco brillante, muy buen conductor del calor y la electricidad, que pertenece a la segunda serie de los elementos de transición y se encuentra nativo en la naturaleza o combinado en forma de sulfuros.

\section{Aplicaciones}

La plata ${ }^{3,4}$ es un material ampliamente utilizado. Sus aplicaciones más frecuentes son en joyería y en la fabricación de vidrio y espejos, fotografía/radiografía, fabricación de baterías, amalgama dental, tintas y aplicaciones médicas (se han usado compuestos de plata para indicaciones tales como irrigaciones vesicales, uretritis, gotas nasales, ulceraciones mucosas, cervicitis, vaginitis, conjuntivitis, etc.). 


\section{Clínica}

La plata no se absorbe por la piel intacta, pero sí lo hace rápidamente por las mucosas, como tal o en formas insolubles (óxido, sulfuro o plata metálica). Así, las sales de plata se absorben bien por vía digestiva o respiratoria y son capaces de precipitar proteínas formando depósitos de color gris azulado/pizarra.

No se conocen efectos agudos por la exposición a la plata, pero sí a algunos compuestos como el óxido y el nitrato de plata, que son irritantes y pueden dar lugar a argiria.

La exposición crónica da lugar a depósitos de forma local o generalizada (argiria), mayoritariamente en las zonas expuestas a la luz solar (principalmente frente, nariz y manos) (fig. 4). Esta pigmentación es gradual.

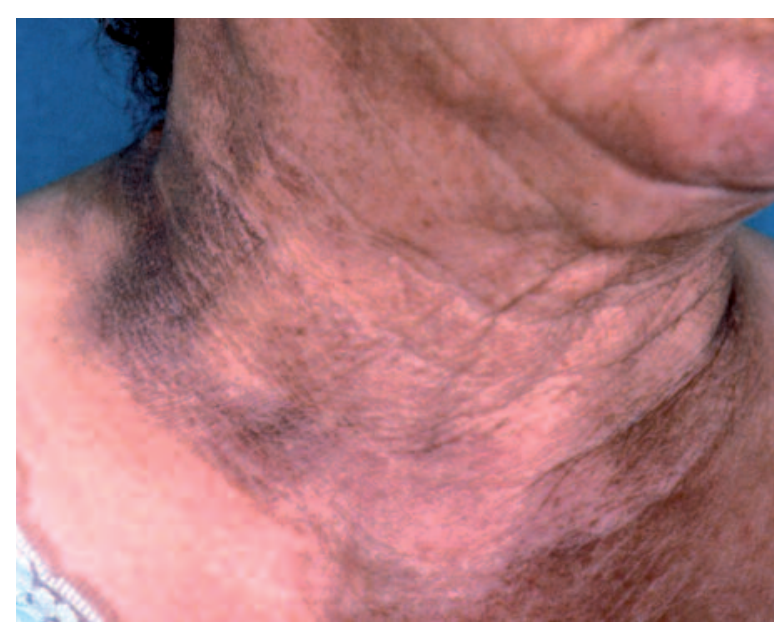

Figura 4. Argiria. Pigmentación grisácea en zonas expuestas por depósito de plata.

Las lesiones locales suelen encontrarse en cara, córnea, uñas (lúnula), cristalino y conjuntiva (argirosis). Pueden aparecer también tatuajes en vías respiratorias.

Las lesiones generalizadas pueden llegar a extenderse a toda la piel y dar lugar a una pigmentación generalizada de color pizarra que ha llevado a denominar a estos individuos «hombres azules» o blue men.
La toxicidad sistémica de la plata es muy escasa excepto en casos de envenenamiento agudo, en los que pueden producirse lesiones intestinales erosivas y hemorrágicas ${ }^{19}$.

\section{BIBLIOGRAFÍA}

1. De la Fuente JA. La biología en la Antigüedad y la Edad Media. 1. ․ㅡ ed. Salamanca: Ediciones Universidad Salamanca; 2002.

2. Sánchez González MA. Historia de la Medicina y humanidades médicas. Barcelona: Elsevier Masson; 2012.

3. Dreisbach T. Bev-Lorraine. Manual de toxicología clínica. 7.a ed. México: Manual Moderno; 2003.

4. Ladrón de Guevara J, Moya Pueyo V. Toxicología médica clínica y laboral. Nueva York: Interamericana McGraw-Hill; 1995.

5. Repetto M. Toxicología fundamental. Madrid: Editorial Díaz de Santos; 1998.

6. Foy F. Curso de materia médica o de farmacología. Barcelona: J. Verdaguer; 1838.

7. Gilbert SG. A small dose of toxicology. The health effects of common chemicals. London: Tylor \& Francis e-Library; 2005.

8. Antommarchi F. Últimos momentos de Napoleón: conclusión del «Diario de Santa-Helena». Barcelona: Imprenta Oliva; 1835.

9. López Mato O. Después del entierro: a veces la muerte no es el final de la historia, sino el comienzo. Buenos Aires: Editorial Sudamericana; 2008.

10. Jourdan AJL. Farmacopea universal. Vol. 3. Madrid: Imprenta de don Ramón Vergés; 1829. p. 402-47.

11. Eisinger J. Was Beethoven lead-poisoned? Beethoven J. 2008; 23(1):15-7.

12. Ferrer A. Intoxicación por metales. An Sist Sanit Navar. 2003;26(1):141-53.

13. Poma PA. Intoxicación por plomo en humanos. An Fac Med Lima [online]. 2008;69(2). [Fecha de consulta: 24-04-2014.] Disponible en: http://www.scielo.org.pe/scielo.php?pid= S1025-55832008000200011\&script=sci_arttext

14. Sanín LH, González-Cossío T, Romieu I, Hernández-Ávila M. Acumulación de plomo en hueso y sus efectos en la salud. Salud Pública Mex [online]. 1998;40(4). [Fecha de consulta: 24-04-2014.] Disponible en: http://www.scielosp.org/scielo.php?script=sci_arttext\&pid=S0036-36341998000400009

15. Academia Nacional de Medicina. Seminario internacional sobre clínica del mercurio. Memorias. Bogotá: Editorial Kimpress; 2006.

16. Carroll L. Alicia en el país de las maravillas. Madrid: Alianza Editorial; 2010.

17. Morales Hernández J, Fleta Zaragozano J, Ayerza Casas A, de Diego Pericas V, Quevedo Sánchez E, Yécora Navarro MD, et al. Baboon syndrome. An Pediatr. 2008;68:486-9.

18. Sánchez-Morillas L, Reaño Martos M, Rodríguez Mosquera M, Iglesias Cadarso C, González Sánchez L, Domínguez Lázaro AR. Síndrome de Baboon. Allergol Immunopathol. 2004;32: 43-5.

19. Fariña MC, Escalonilla P, Grilli R, Soriano ML, Martín L, Requena $L$, et al. Argiria generalizada secundaria a la administración tópica de nitrato de plata. Actas Dermosifiliogr. 1998;89(10): 547-52. 